EESTI NSV TEADUSTE AKADEEMIA TOIMETISED. XI KOIDE FOUSIKALIS-MATEMAATIEISTE JA TEHNILISTE TEADUSTE SEERIA. 1962, NR. 3

ИЗВЕСТИЯ АКАДЕМИИ НАУК ЭСТОНСКОИ ССР. ТОМ ХІ СЕРИЯ ФИЗИКО-МАТЕМАТИЧЕСКИХ И ТЕХНИЧЕСКИХ НАУК. 1962, № 3

\title{
ОБ ОДНОМ ВАРИАЦИОННОМ МЕТОДЕ ПРИБЛИЖЕННОГО РЕШЕНИЯ ДИФФЕРЕНЦИАЛЬНЫХ УРАВНЕНИЙ С ЧАСТНЫМИ ПРОИЗВОДНЫМИ
}

\author{
л. АИНОЛА,
}

кандидат физико-математических наук

Для приближенного решения дифференциальных уравнений є частными производными общеизвестны вариационные методы Ритца и Канторовича. При вариационном методе Ритца решение задачи ищется в виде ряда, члены которого как функции задаются, а постоянные коэффициенты определяются из системы алгебраических уравнений, являющейся условием стационарности соответствующего функционала. При методе Канторовича коэффициентами ряда являются функции от одной переменной, которые определяются из системы обыкновенных дифференциальных уравнений. В настоящей заметке предлагается вариационный метод, где члены ряда ищутся в виде произведения двух неизвестных сомножителей, один из которых является функцией одной переменной, а другой - функцией другой переменной. Для их определения, правилами вариационного исчисления, получаются две системы обыкновенных дифференциальных уравнений. При этом коэффициенты одной системы зависят от решения другой системы и наоборот. Если удастся эти системы с неопределенными коэффициентами решить, то значения этих постоянных коэффициентов можно определить из системы трансцендентных уравнений. Принципиальное отличие предлагаемого метода от методов Ритца и Канторовича состоится в том, что здесь проводится одновременное варьирование в двух направлениях. Аналогичная идея использована при приближенном методе Харти-Фока в квантовой механике [ $\left.{ }^{1}\right]$ й при методе Вайнштейна $\left.{ }^{2}\right]$ для приближенного разделения переменных.

\section{1. Описание метода}

Рассмотрим задачу стационарности функционала

$$
I=\iint_{D} F\left(x, y, u, u_{x}, u_{y}, u_{x x}, \ldots\right) d x d y
$$

где $D$ прямоугольная область. 
Необходимым условием стационарности функционала является дифференциальное уравнение с частными производными

$$
H(u) \equiv F_{u}-\frac{\partial}{\partial x} F_{u_{x}}-\frac{\partial}{\partial y} F_{u_{y}}+\frac{\partial 2}{\partial x^{2}} F_{u_{x x}}+\ldots=0 .
$$

Пусть $F(x, y, u, \ldots)$ имеет такую форму, что множители перед неизвестной функцией и его производными в уравнении (1.2) разделяются на произведения функции от $x$ и функции от $y$. Пусть краевые условия для $u(x, y)$ на контуре $x=x_{1}, x=x_{2}, y=y_{1}, y=y_{2}$, ограничива́юшем область $D$, будут однородными.

Ищем приближенное решение уравнения (1.2) в виде

$$
u(x, y)=\sum_{i=1}^{n} f_{i}(x) g_{i}(y)
$$

где каждая слагаемая удовлетворяет краевым условиям.

Функции $f_{i}(x)$ и $g_{i}(y)$ определяются из условия, что первая вариация функционала (1.1) равняется нулю

$$
\delta I=0 .
$$

Подставим выражение (1.3) в функционал (1.1)

$\boldsymbol{I}=\int_{D} \int_{D} G\left(x, y, f_{1}, f_{1}^{\prime}, f_{1}^{\prime \prime}, \ldots, f_{n}, f_{n}^{\prime}, \ldots, g_{1}, g_{1}, \ldots, g_{n}, g_{n} \ldots\right) d x d y$

Здесь штрихами обозначено дифференцирование по координате $x$ и точками - по координате $y$.

Так как

$$
\delta u=\sum_{i=1}^{n}\left(g_{i} \delta f_{i}+f_{i} \delta g_{i}\right)
$$

то в вариационном исчислении известным путем получим, что первая вариация этого функционала имеет вид

$$
\begin{aligned}
& \delta I=\sum_{i=1}^{n}\left\{\int_{x_{1}}^{x_{2}} L_{i} \delta f_{i} d x+\int_{y_{1}}^{y_{2}} K_{i} \delta g_{i} d y+\right. \\
& +\left.\left(M_{1 i} \delta f_{i}+M_{2 i} \delta f_{1}^{\prime}+\ldots\right)\right|_{\begin{array}{l}
x=x_{2} \\
x=x_{1}
\end{array}}+ \\
& \left.+\left(N_{1 i} \delta g_{i}+N_{2 i} \delta g_{i}+\ldots\right) \mid \begin{array}{l}
y=y_{2} \\
y=y_{1}
\end{array}\right\} .
\end{aligned}
$$

Здесь

$$
\begin{aligned}
& L_{i} \equiv \int_{y_{1}}^{y_{2}} \frac{\partial G}{\partial f_{i}} d y-\frac{d}{d x} \int_{v_{1}}^{y_{2}} \frac{\partial G}{\partial f_{i}^{\prime}} d y+\frac{d^{2}}{d x^{2}} \int_{v_{1}}^{y_{2}} \frac{\partial G}{\partial f_{i}^{\prime \prime}} d y-\ldots \\
& K_{i} \equiv \int_{x_{1}}^{x_{2}} \frac{\partial G}{\partial g_{i}} d x-\frac{d}{d y} \int_{x_{1}}^{x_{2}} \frac{\partial G}{\partial g_{i}} d x+\frac{d^{2}}{d y^{2}} \int_{x_{1}}^{x_{2}} \frac{\partial G}{\partial g_{i}} d x-\ldots
\end{aligned}
$$


Предполагая, что краевыми условиями, наложенными на $u(x, y)$, определяются значения $f_{i}\left(x_{1}\right), f_{i}^{\prime}\left(x_{1}\right), \ldots, f_{i}\left(x_{2}\right), f_{i}^{\prime}\left(x_{2}\right), \ldots$ и $g_{i}\left(y_{1}\right), g_{i}\left(y_{1}\right), \ldots, g_{i}\left(y_{2}\right), g_{i}^{\prime}\left(y_{2}\right), \ldots$, получим, что $\delta f_{i}=\delta f_{i}^{\prime}=\ldots=0$ при $x=x_{1}$ и $x=x_{2}$, а также $\delta g_{i}=\delta g_{i}=\ldots=0$ при $y=y_{1}$ и $y=y_{2}$. Из условия (1.4) и выражения (1.7) теперь вытекают следующие системы для определения функщий $f_{i}(x)$ и $g_{i}(y)$

$$
L_{i}=0, \quad K_{i}=0 \quad(i=1,2, \ldots, n) .
$$

Далее надо решить эту систему (точно или приближенно), рассматривая интегралы от искомых функций, имеющиеся в уравнениях, как неопределенные постоянные коэффициенты. Для определения этих коэффициентов надо полученные решения и их производные подставить в соответствующие выражения интегралов и произвести интегрирование. В результате получается система уравнений относительно коэффиниентов.

В частном случае, если задавать функции $f_{i}(x)$ или $g_{i}(y)$, описанный метод совпадает с методом Канторовича.

Отметим, что существует возможность применять аппроксимацию (1.3) в методе Галеркина. В этом случае функции $f_{i}(x)$ и $g_{i}(y)$ определяются из системы

$$
\iint_{D} H f_{i} d x d y=0, \quad \iint_{D} H g_{i} d x d y=0 .
$$

Для более подробного пояснения метода рассмотрим простую конкретную задачу.

\section{2. Пример}

Рассмотрим задачу решения уравнения Пуассона

$$
u_{x i}+u_{y y}+p(x) q(y)=0
$$

при нулевых граничных условиях

$$
u(x, y)=0
$$

на прямолинейных краях $x= \pm a, y= \pm b$.

Эта задача эквивалентна задаче о минимуме функционала

$$
I=\int_{D} \int\left(u_{x}^{2}+u_{y}^{2}-2 p q\right) d x d y .
$$

Выразим $u(x, y)$ в виде (1.3) и предположим, что $f_{i}(x)$ и $g_{i}(y)$ удовлетворяют граничным условиям

$$
f_{i}( \pm a)=g_{i}( \pm b)=0,
$$

вытекающим из (2.2). 
Тогда из вариации функционала (2.3)

$$
\delta I=\iint_{D} \sum_{k=1}^{n}\left[\sum_{i=1}^{n}\left(f_{i}^{\prime \prime} g_{i}+f_{i} g_{i}\right)+p q\right]\left(g_{k} \delta f_{k}+f_{k} \delta g_{k}\right) d x d y
$$

получим следующие дифференциальные уравнения

$$
\begin{aligned}
& \sum_{i=1}^{n}\left(a_{i k}^{(1)} f_{i}^{\prime \prime}-a_{i k}^{(2)} f_{i}\right)+a_{k}^{(3)} p=0, \\
& \sum_{i=1}^{n}\left(\beta_{i k}^{(1)} g_{i}-\beta_{i k}^{(2)} g_{i}\right) !+\beta_{k}^{(3)} q=0, \\
& (k=1,2, \ldots, n) .
\end{aligned}
$$

Здесь коэффициенты обозначены следующим образом:

$$
\begin{aligned}
& a_{i k}^{(1)}=\int_{-b}^{b} g_{i} g_{k} d y, \quad a_{i k}^{(2)}=\int_{-b}^{b} g_{i} g_{k} d y, \quad a_{k}^{(3)}=\int_{-b}^{b} g_{k} q d y, \\
& \beta_{i k}^{(1)}=\int_{-a}^{a} f_{i} f_{k} d x, \quad \beta_{i k}^{(2)}=\int_{-a}^{a} f_{i}^{\prime} f_{k}^{\prime} d x, \quad \beta_{k}^{(3)}=\int_{-a}^{a} f_{k} p d x .
\end{aligned}
$$

Решения систем (2.6) при граничных условиях (2.4) будут

$$
\begin{aligned}
& f_{i}=f_{i}\left(x ; \alpha_{s t}^{(1)}, \alpha_{s t}^{(2)}, \alpha_{s}^{(3)}\right), \\
& g_{i}=g_{i}\left(y ; \beta_{s t}^{(1)}, \quad \beta_{s t}^{(2)}, \quad \beta_{s}^{(3)}\right) .
\end{aligned}
$$

Используя эти функции получим из (2.7) системы

$$
\begin{aligned}
& \alpha_{i k}^{(r)}=A_{i k}^{(r)}\left(\beta_{s t}^{(1)}, \beta_{s t}^{(2)}, \beta_{s}^{(3)}\right), \\
& \beta_{i k}^{(r)}=B_{i k}^{(r)}\left(\alpha_{s t}^{(1)}, \alpha_{s t}^{(2)}, \alpha_{s}^{(3)}\right), \\
& (r=1,2,3),
\end{aligned}
$$

определяющие неизвестные коэффициенты.

\section{3. Численные результаты}

Приведем численные результаты решения рассмотренной задачи в самом простом случае.

Пусть $p(x)=1, q(y)=1$ и $n=1$. Используя обозначения.

$$
a_{1}^{2}=\frac{a_{11}^{(2)}}{a_{11}^{(1)}} a^{2}, \quad a_{2}=\frac{a_{1}^{(3)}}{a_{11}^{(2)}}, \quad \beta_{1}^{2}=\frac{\beta_{11}^{(2)}}{\beta_{11}^{(1)}} b^{2}, \quad \beta_{2}=\frac{\beta_{1}^{(3)}}{\beta_{11}^{(2)}},
$$


получим. что функции (2.8) имеют вид

$$
\begin{aligned}
& f_{1}=\alpha_{2}\left(1-\frac{\operatorname{ch} \alpha_{1} \frac{x}{a}}{\operatorname{ch} \alpha_{1}}\right), \\
& g_{1}=\beta_{2}\left(1-\frac{\operatorname{ch} \beta_{1} \frac{y}{b}}{\operatorname{ch} \beta_{1}}\right) .
\end{aligned}
$$

Система (2.9) для определения коэффициентов будет

$$
\begin{aligned}
& \alpha_{1}^{2}=\frac{\beta_{1}^{2}\left(\beta_{1} \text { th }^{2} \beta_{1}+\text { th } \beta_{1}-\beta_{1}\right) a^{2}}{\left(3 \beta_{1}-3 \text { th } \beta_{1}-\beta_{1} \text { th }^{2} \beta_{1}\right) b^{2}}, \\
& \alpha_{2}=\frac{2\left(\beta_{1}-\text { th } \beta_{1}\right) b^{2}}{\beta_{1}^{2} \beta_{2}\left(\beta_{1} \text { th }^{2} \beta_{1}+\text { th } \beta_{1}-\beta_{1}\right)}, \\
& \beta_{1}^{2}=\frac{a_{1}^{2}\left(\alpha_{1} \text { th }^{2} \alpha_{1}+\text { th } \alpha_{1}-\alpha_{1}\right) b^{2}}{\left(3 \alpha_{1}-3 \text { th } \alpha_{1}-\alpha_{1} \text { th }^{2} \alpha_{1}\right) a^{2}}, \\
& \beta_{2}=\frac{2\left(\alpha_{1}-\text { th } \alpha_{1}\right) a^{2}}{a_{1}^{2} \alpha_{2}\left(\alpha_{1} \text { th }^{2} \alpha_{1}+\text { th } \alpha_{1}-\alpha_{1}\right)} .
\end{aligned}
$$

При $a=2 b$ получаемое решение имеет вид

$$
u(x, y)=1,77456\left(1-\frac{\operatorname{ch~} 1,58713 \frac{x}{b}}{\operatorname{ch} 3,17426}\right)\left(1-\frac{\operatorname{ch~} 0,85201 \frac{y}{b}}{\operatorname{ch~} 0,85201}\right) b^{2}
$$

Метод Канторовича с $g_{1}(y)=b^{2}-y^{2}$ дает решение [3]

$$
u(x, y)=\frac{1}{2}\left(b^{2}-y^{2}\right)\left(1-\frac{\operatorname{ch~} 1,58114 \frac{x}{b}}{\operatorname{ch} 3,16228}\right) \text {. }
$$

При $b=1, x=0$ и $y=0$ решение (3.4) принимает значение $u(0,0)=$ $=0,45248$, точное решение [3] 0,45549. Решение Канторовича (3.5) дает 0,45721 . При $b=1, x=1, y=\frac{1}{2}$ соответственно получается $u\left(1, \frac{1}{2}\right)=$ $=0,29581 ; 0,29501$ и 0,29532 .

\section{Л И Т Е Р А Т У Р А}

1. Г. Бете и Э. Солпи тер, Квантовая механика атомов с одним и двумя электронами, М., 1960, $\$ 30$ и 31 .

2. Л. А. В а й н ш те й н, Метод приближенного разделения переменных и его применение к граничным задачам электродинамики и акустикн, Ж. техн. физ., т. 27, B. $9,2109-2128$.

3. Л. В. К анторович и В. И. Крылов, Приближенные методы высшего анализа, М.-Л., 1952 , гл. $4, \S 3$. 


\section{OHEST LIGIKAUDSEST OSATULETISTEGA DIFERENTSIAALVORRANDITE LAHENDAMISE VARIATSIOONMEETODIST}

\section{Ainola}

\section{Resümee}

Rohkesti kasutatavate Ritzi ja Kantorovitši variatsioonmeetodite puhul aproksimeeritakse lahend sellise rea kujul, mille iga liige sisaldab varieeritavat konstanti või ühemuutuja funktsiooni. Käesolevas uurimuses esitatakse meetod, kus aproksimeeritavate funktsioonide varieerimine toimub mōlema koordinaadi suunas. Selleks antakse lahend ette erinevate argumentidega ühemuutuja funktsioonide korrutiste summana (1.3). Variatsioonarvutuse kasutamisega saadakse korrutatavate funktsioonide määramiseks kaks harilike diferentsiaalvõrrandite süsteemi (2.6), kus esimese süsteemi kordajad-koefitsiendid (2.7) sōltuvad teise süsteemi lahendist ja vastupidi. Kui süsteemi $(2.6)$ lahend $(2.8,3.2)$ on teada, määratakse koefitsiendid harilike võrrandite süsteemist $(2.9,3.3)$.

\section{Eesti NSV Teaduste Akadeemia} Küberneetika Instituut
Saabus toimetusse

15. I 1962

\section{AN APPROXIMATE VARIATIONAL MEFHOD FOR SOLVING PARTIAL DIFFERENTIAL EQUATIONS}

L. Ainola

\section{Summary}

By the well-known variational methods of Ritz and Kantorovitch the solution of a partial differential equation is approximated by the series with each term involving an indeterminate constant or function of one variable. In the present note a method is proposed in which the functions approximated are variated in directions of both coordinates. For this, the terms of series are taken as products of two functions of different coordinates (1.3). These functions are determined from two systems of ordinary differential equations (2.6) obtainet by the variational calculus. The coefficients of the first system (2.7) are dependent on the solution of the second system, and vice versa. If solutions of systems $(2.8,3.2)$ are found, the coefficients are determined from the system of common equations $(2.9,3.3)$.

Academy of Sciences of the Estonian S.S.R., Institute of Cybernetics

Received Jan. 15th, 1962 